\title{
Laura Costantino
}

orcid: 0000-0002-7563-3537

\section{Politiche europee e nazionali di contrasto allo spreco alimentare nella produzione primaria: analisi e prospettive future}

1. In seguito all'adozione da parte dell'Assemblea Generale delle Nazioni Unite della Risoluzione "Transforming our world: the 2030 Agenda for Sustainable Development", il Parlamento europeo ha adottato la Risoluzione sull'iniziativa sull'efficienza sotto il profilo delle risorse: ridurre lo spreco alimentare, migliorare la sicurezza alimentare (Risoluzione del 16 maggio 2017), aggiornando gli obiettivi in materia di contrasto allo spreco di cibo, già indicati nella precedente Risoluzione del 2012 (Risoluzione del 19 gennaio 2012 su Come evitare lo spreco di alimenti: strategie per migliorare l'efficienza della catena alimentare nell'UE), in risposta all'obiettivo 12.3 del documento Agenda 2030. In particolare, a fronte delle stime delle Nazioni Unite che riferiscono dell'incremento della popolazione mondiale che patisce la fame, il documento Agenda 2030 individua l'obiettivo di ridurre del $50 \%$ entro il 2030 gli sprechi alimentari globali pro capite a livello di vendita al dettaglio e da parte dei consumatori, e di ridurre le perdite alimentari lungo le catene di produzione e di approvvigionamento, ivi comprese le perdite durante la fase della produzione primaria, del trasporto e dello stoccaggio. Appare subito evidente dalla strutturazione degli obiettivi proposti che la problematica degli sprechi alimentari coinvolge tutta la filiera, dalla produzione agricola fino al consumo, e dunque si presenta come un tema estremamente complesso, comportando l'individuazione di differenti sistemi di intervento (normativo) a seconda della specifica fase produttiva sulla quale si interviene. Allo stesso tempo, differenti sono le cause che conducono alla creazione di sprechi, a loro volta molto diverse per ogni specifico comparto produttivo. 
2. L'Unione europea ha da tempo mostrato attenzione al tema della riduzione degli sprechi alimentari, in vista del raggiungimento di molteplici obiettivi, tutti estremamente ambiziosi, quali la riduzione dei rifiuti alimentari anche in considerazione dell'impatto degli stessi sul surriscaldamento globale; la gestione razionale delle risorse naturali utilizzate per la produzione di cibo; la drastica riduzione dei nuovi poveri, ovvero di quella fascia di popolazione europea in graduale crescita che non ha accesso costante al cibo (il tema è strettamente legato al riemergere della nuova food insecurity); la ricerca di una migliore sostenibilità sia economica che ambientale della filiera agroalimentare, anche in vista della necessità di assicurare un reddito adeguato ai produttori agricoli ${ }^{1}$.

L'attuale emergere in termini così dirompenti del fenomeno dello spreco alimentare non è casuale, ma strettamente connesso alla graduale scelta politico-economica dell'UE volta a virare gli interventi di governo del sistema di produzione alimentare, sganciandoli dalle misure interventiste che hanno caratterizzato il primo periodo della $\mathrm{PAC}^{2}$. In un sistema economico nel quale non sono più presenti misure di intervento sulla formazione dei prezzi né sull'andamento delle produzioni agroalimentari, la sostenibilità delle filiere produttive è lasciata al libero gioco del mercato e, dunque, la presenza di sprechi alimentari rappresenta una inevitabile conseguenza se si considerano le peculiarità e specificità del sistema agroalimentare rispetto ad altri settori di mercato.

L'agricoltura è tuttora oggetto di attenzione da parte della politica europea in termini multifunzionali: gli agricoltori sono i primi custodi dell'ambiente rurale e infatti si riconosce all'agricoltura un ruolo chiave e determinante nella gestione efficiente e razionale delle risorse naturali e, più in generale, nella costruzione dello sviluppo sostenibile in termini economici, ambientali e sociali ${ }^{3}$.

${ }^{1}$ L'attenzione al tema dello spreco alimentare nelle politiche europee è resa esplicita, oltre che nelle due Risoluzioni citate in precedenza, nelle Conclusioni del Consiglio UE 10730/16 del 28 giugno 2016, Perdite e sprechi alimentari, e si inserisce, in una prospettiva di insieme, nel contesto delle misure europee relative all'economia circolare approdate nell'adozione della proposta di direttiva sui rifiuti (COM/2014/397 final).

${ }^{2}$ Sull'evoluzione della politica agricola comune la bibliografia è vastissima; si rinvia, in questa sede, ai fondamentali contributi di L. Costato (a cura di), Trattato breve di diritto agrario italiano e comunitario, Padova 2003; Id., La controriforma della PAC, "Rivista di Diritto Agrario" 2010, I, p. 369; Id., Agricoltura, ambiente e alimentazione nell'evoluzione del diritto dell'Unione Europea, "Rivista di Diritto Agrario" 2015, p. 210; Id., Diritto alimentare e diritto al cibo: dal I Piano Mansholt alla Riforma del 2013, "Rivista di Diritto Agrario" 2015, p. 306; Id., Per una storia della PAC (a sessant'anni dall 'inserimento dell'agricoltura nel progetto di Trattato CEE), "Rivista di Diritto Agrario" 2017, p. 64.

${ }^{3}$ Comunicazione della Commissione COM(2017) 713 final, del 29/11/2017, Il futuro dell'alimentazione e dell'agricoltura: “ (...) Gli agricoltori dell'UE sono i primi custodi dell'ambiente naturale, in quanto curano le risorse del suolo, dell'acqua, dell'aria e della biodiversità (...) Nessuno 
Allo stesso tempo, si evidenzia, però, che la legislazione europea ha solo di recente posto le basi per la progressiva realizzazione di una disciplina che tuteli le imprese agricole dalle asimmetrie del potere negoziale rispetto agli acquirenti dei prodotti agricoli.

A ben guardare, la filiera agroalimentare presenta, infatti, una specifica debolezza strutturale che la caratterizza e la distingue dalle altre filiere produttive e che risiede in primo luogo nella asimmetria tra produzione agricola e industria alimentare ${ }^{4}$.

Le imprese agricole sono strutturalmente dipendenti dalle imprese acquirenti del prodotto, a causa sia della peculiarità dell'attività agricola che implica la dipendenza nei tempi e nella quantità di produzione da variabili incontrollabili, sia della strutturale frammentazione dell'offerta produttiva nei mercati europei. Le istituzioni europee hanno da qualche tempo cercato di affrontare le problematiche specifiche delle filiere agroalimentari: basti pensare alla Comunicazione della Commissione sul Miglior funzionamento della filiera alimentare in Europa (COM 2009591 def.). Il documento nasce dalla preoccupazione per la fluttuazione dei prezzi al consumo a forte discapito delle ragioni economiche dei consumatori e degli imprenditori agricoli. L'utilizzo di pratiche commerciali sleali tra imprese e l'instabilità dei prezzi dei prodotti alimentari e delle materie prime agricole rappresentano le due principali preoccupazioni dell'azione dell'Unione, in quanto comportano una serie di problematiche strutturali che indeboliscono la filiera. Una di queste è rappresentata dal crescente impoverimento dei produttori agricoli, a fronte di un costante aumento del margine di guadagno delle imprese di trasformazione e dei commercianti all'ingrosso. Al fine di cercare una risposta normativa adeguata all'esigenza di tutela del reddito dei produttori agricoli, si sottolinea l'importanza di una maggiore trasparenza sulla formazione dei prezzi lungo la filiera e di interventi correttivi sull'abuso di posizione dominante da parte di alcuni attori economici operanti lungo la filiera agroalimentare (imprese fornitrici di fattori produttivi agli imprenditori agricoli, imprese di trasformazione, grande distribuzione organizzata) e più in

di questi benefici può tuttavia essere dato per scontato: a differenza della maggior parte degli altri settori economici, l'agricoltura è fortemente influenzata dalle condizioni meteorologiche; è spesso messa a dura prova dalla volatilità dei prezzi, da calamità naturali, parassiti e malattie (...) il settore si trova ad affrontare problemi di bassa redditività (dovuta anche agli elevati standard di produzione dell'UE), di costo elevato dei fattori di produzione e della frammentarietà del settore primario. (...) la PAC può contribuire a ridurre gli sprechi alimentari e le perdite alimentari incoraggiando migliori pratiche di produzione e lavorazione (...), sostenendo iniziative che trasformano i tradizionali schemi di consumo produzione-uso-eliminazione in una bioeconomia circolare".

${ }^{4}$ A. Iannarelli, Le relazioni contrattuali tra agricoltura e industria nella recente esperienza giuridica italiana, in: Diritto agrario e società industriale, t. II, Il sistema agroalimentare, Bari 1993; Id., Il diritto dell'agricoltura nell'era della globalizzazione, Bari 2003. 
generale si evidenzia che la presenza di situazioni di squilibrio del potere negoziale è la prima fondamentale causa dell'impoverimento dei produttori agricoli. Lo squilibrio nel potere negoziale lungo la catena agroalimentare è al centro di attenzione di una distinta Risoluzione del Parlamento europeo che fa luce sulla diffusione di pratiche commerciali sleali quali, ad esempio, pagamenti tardivi, modifiche unilaterali dei contratti, mancanza di informazione sulla formazione dei prezzi, abusi di potere di mercato ${ }^{5}$.

La conseguenza dell'asimmetria del potere negoziale è senza dubbio il rischio di messa in atto di pratiche commerciali sleali, ma anche l'uscita dalla filiera di tutti quei prodotti che non corrispondono alle precise indicazioni qualitative imposte dall'acquirente nei contratti con l'impresa agricola. Inoltre, l'asimmetria negoziale ha precise ripercussioni sulla competitività della filiera agroalimentare, con la duplice conseguenza di abbassare i profitti delle imprese agricole, limitandone la capacità di crescita e di innovazione, sia nella qualità dei prodotti che nei processi produttivi. Fino a quando la filiera agroalimentare non raggiungerà un equilibrio nelle relazioni contrattuali tra gli operatori economici coinvolti garantendo prezzi equi e competitività, il reddito degli imprenditori agricoli sarà iniquo, la produzione agricola sarà spesso eccedentaria rispetto alla domanda delle grandi imprese di trasformazione o di vendita al dettaglio, causando fluttuazione dei prezzi a creazione di sprechi. Nella Risoluzione del Parlamento europeo del 16 maggio 2017 sull'iniziativa sull'efficienza sotto il profilo delle risorse: ridurre lo spreco alimentare, migliorare la sicurezza alimentare, ai punti 86 e 87 , si evidenzia espressamente la stretta connessione tra pratiche commerciali sleali nell'ambito della catena di approvvigionamento e sprechi alimentari ${ }^{6}$.

Superare le problematiche derivanti dalle situazioni di squilibrio del potere negoziale attraverso la regolamentazione delle condotte commerciali sleali è condizione indispensabile per il raggiungimento dell'obiettivo di efficienza e sostenibilità delle filiere agroalimentari. È di recentissima pubblicazione la proposta di direttiva in materia di pratiche commerciali sleali nei rapporti tra

${ }^{5}$ Risoluzione del Parlamento europeo del 19 gennaio 2012 sugli squilibri della catena di distribuzione alimentare.

6 “'(...) le pratiche commerciali sleali nell'ambito della catena di approvvigionamento possono generare sprechi di alimenti; (...) la soluzione del problema delle pratiche sleali migliorerà la posizione degli agricoltori, l'anello più debole della catena, e, inoltre, riducendo la sovrapproduzione e l'accumulo di eccedenze, potrà aiutare non solo a stabilizzare i prezzi e a offrire agli agricoltori prezzi (...) equi e remunerativi, ma anche a ridurre sia gli sprechi alimentari nell'intera filiera sia le perdite generate nelle aziende agricole familiari; (...) una più equa retribuzione dei produttori conferirebbe maggiore valore ai prodotti determinando una diminuzione degli sprechi alimentari negli anelli finali della catena di approvvigionamento". 
imprese nella filiera alimentare ${ }^{7}$, che mira a ridurre le pratiche commerciali sleali nella filiera alimentare mediante l'introduzione di un livello minimo di tutela comune a tutta l'UE, comprendente un breve elenco specifico di pratiche commerciali sleali vietate. La tutela è rivolta ai fornitori di piccole e medie dimensioni della filiera alimentare, nelle relazioni contrattuali con imprese di grandi dimensioni. L'obiettivo è quello di contribuire ad assicurare un tenore di vita equo alla popolazione agricola, realizzando in tal modo uno degli obiettivi della politica agricola comune ai sensi dell'articolo 39 del TFUE.

Le problematiche specifiche della filiera agroalimentare sono tra loro tutte connesse e necessitano di un approccio globale e di sistema che ruoti intorno alla tutela del bene alimento.

L'importante produzione di spreco alimentare incide, infatti, direttamente su alcuni principi giuridicamente tutelati dalla legislazione europea, ed in particolare dall'art. 39 TFUE.

Gli obiettivi della PAC consistono nell'incremento della produttività in agricoltura attraverso il progresso tecnico e l'impiego ottimale dei fattori di produzione; nell'assicurare un tenore di vita equo alla popolazione agricola; nella stabilizzazione dei mercati; nella garanzia della sicurezza degli approvvigionamenti; ed infine nell'assicurare prezzi ragionevoli per i consumatori. L'art. 11 TFUE impone alle politiche europee la tutela dell'ambiente nella prospettiva della promozione dello sviluppo sostenibile.

Lo spreco alimentare rappresenta una violazione dei principi giuridici alla base della politica agricola ed ambientale europea. L'obiettivo dell'incremento della produttività nel settore agricolo non può non comportare interventi volti ad arginare la perdita dei prodotti agricoli non raccolti o invenduti, anche al fine di un uso sostenibile dei fattori produttivi impiegati. Direttamente dipendente dalla perdita di cibo nella fase della produzione agricola è la mancata remunerazione degli agricoltori.

Un'azione politica contro lo spreco alimentare dovrà affrontare problematiche diversificate ma tutte specifiche del sistema cibo e il tema dello spreco può rappresentare un'occasione per le istituzioni europee di guardare alla filiera agroalimentare in modo globale. Il fenomeno dello spreco non è altro, infatti, che un indice dell'inefficienza della filiera, non solo da un punto di vista meramente economico ma anche nell'ottica degli obiettivi fatti propri dalle recenti politiche di sostenibilità ed uso efficiente delle risorse naturali.

3. L'approccio globale alla filiera agroalimentare nell'ottica della ricerca di una risposta normativa allo squilibrio nelle relazioni negoziali tra imprese agrico-

\footnotetext{
${ }^{7} \operatorname{COM}(2018) 173$ final.
} 
le ed imprese dell'alimentazione è assunto nel Progetto di legge francese "pour l'équilibre des relations commerciales dans le secteur agricole et alimentaire et une alimentation saine et durable" . Procedendo nella direzione già intrapresa dal legislatore francese con l'adozione della legge n. 2016-138 dell'11 febbraio 2016, "Loi relative à la lutte contre le gaspillage alimentaire", il progetto di legge, che mira ad intervenire sull'equilibrio tra gli attori della filiera, riprende l'obiettivo di contrasto allo spreco alimentare, nella convinzione che "La lutte contre le gaspillage alimentaire s'inscrit pleinement dans un double objectif de mise en valeur des produits et de responsabilisation des acteurs afin d'oeuvrer pour une répartition plus optimale des biens produits", rafforzando allo stesso tempo lo stretto legame tra squilibrio economico e negoziale tra imprese agricole e imprese commerciali, e creazione di sprechi.

Tra i pochi interventi normativi rivolti alla riduzione degli sprechi alimentari, l'Italia ha rappresentato un campo di sperimentazione giuridica interessante. La recente legge n. 166/2016 propone di ridurre gli sprechi per ciascuna delle fasi di produzione, trasformazione, distribuzione e somministrazione di prodotti alimentari, in primo luogo attraverso il recupero e la donazione di "eccedenze" alimentari ${ }^{10}$. In riferimento al contrasto allo spreco lungo la fase della produzione primaria, la legge prevede la possibilità della cessione a titolo gratuito delle "eccedenze" in campo da parte degli imprenditori agricoli, a condizione che le attività di raccolta dei prodotti agricoli avvengano direttamente e sotto la responsabilità dei soggetti donatari, i quali provvederanno alla successiva distribuzione gratuita. L'obiettivo di contrasto allo spreco alimentare attraverso il ricorso a principi di solidarietà sociale, che da tempo rappresenta l'approccio regolativo del legislatore italiano ${ }^{11}$, a nostro avviso, rappresenta una pregevole iniziativa in termini etici e sociali ma, allo stesso tempo, un importante limite. La tematica dello spreco alimentare, infatti, non può risolversi nel ricorso alla redistribuzione gratuita alle persone indigenti; anzi, si rischia,

${ }^{8}$ Progetto di legge n. 627, del 21 aprile 2018.

9 A. Denizot, Les premiers pas hésitants de la lutte contre le gaspillage alimentaire, "RTDCiv." 2016, p. 473; L. Collot, Fin des condamnations pour vol de produits périmés dans les poubelles des supermarchés, "Dalloz actualité" 25 janvier 2016; L. González Vaqué, French and Italian food waste legislation. An example for other EU Member States to follow?, "European food and feed law Review" 2017, n. 3.

${ }^{10}$ I. Trapè, Lo spreco alimentare e la legge italiana n. 166 del 2016, "Rivista di Diritto Agrario", 2017, p. 263; G. Maccioni, Spreco alimentare. Regole e limiti nella transizione verso modelli agroalimentari sostenibili, Torino 2018.

${ }^{11}$ La legge n. 155/2003, nota come legge del buon samaritano, equipara le ONLUS al consumatore finale, ai fini del corretto stato di conservazione, trasporto, deposito e utilizzo degli alimenti, facilitando l'opera di redistribuzione gratuita alimentare dal punto di vista del rispetto della normativa europea sulla sicurezza alimentare. 
in tal modo, di collegare indissolubilmente gli interventi normativi di politica sociale all'utilizzo degli sprechi alimentari ai fini di redistribuzione alimentare gratuita, rendendo paradossalmente necessaria la produzione di sprechi alimentari in quanto utile strumento per il soddisfacimento degli obiettivi di politica sociale. La lotta allo spreco alimentare nella prospettiva di politica europea assume un respiro più ampio, in una dimensione complessiva che guarda all'efficienza di tutta la filiera agroalimentare. Si assiste, dunque, ad una non perfetta coincidenza in termini di obiettivi politici tra l'approccio regolativo europeo e l'intervento normativo italiano in materia di sprechi alimentari.

\title{
THE EU AND THE NATIONAL POLICY TO COMBAT FOOD WASTE IN PRIMARY PRODUCTION: AN ANALYSIS AND FUTURE PROSPECTS
}

\author{
Sum mary
}

Following the adoption by the United Nations General Assembly of the Resolution "Transforming our world: the 2030 Agenda for Sustainable Development," the European Parliament adopted a resolution initiating resource efficiency aimed to reduce food waste and to improve food security (Resolution of 16 May 2017), updating the objectives on food waste, already singled out in the previous Resolution of 2012 (Resolution of 19 January 2012 on How to avoid food waste: strategies for improving the efficiency of the food chain in the EU), in response to Objective 12.3 of the Agenda 2030 document. In particular, in view of the United Nations estimates referring to the increase in the world's hunger population, the Agenda 2030 document identifies the goal of reducing food wastage per person by $50 \%$ by the end of 2030 and aims at reducing food losses along production and supply chains, as well as losses during the primary production, the transport and the storage of goods.

This paper looks at the actions which the European Union has undertaken in order to achieve the objective of reducing food waste, with particular regard to the reduction of food losses along primary production. The European legislative intervention intended to reduce food waste at the primary production level in particular, will necessarily have to deal with the specific problems of agricultural enterprises operating along the supply chain, such as the specific contractual weakness suffered by food producers against the agricultural products purchasers. The regulatory solutions implemented to facilitate the reduction of food losses at the primary production level cannot, in fact, be limited to the proposition of food donations; the reduction of waste during primary production is closely connected with a wider problem which is equitable remuneration of agricultural producers (Article 39 TFEU) if we consider the obvious impact which a loss of perfectly marketable agricultural products has on their earning resulting from the agricultural activity in which they are involved. 


\title{
POLITICHE EUROPEE E NAZIONALI DI CONTRASTO ALLO SPRECO ALIMENTARE NELLA PRODUZIONE PRIMARIA: ANALISI E PROSPETTIVE FUTURE
}

\author{
Riassunto
}

In seguito all'adozione da parte dell'Assemblea Generale delle Nazioni Unite della Risoluzione "Trasformare il nostro mondo. L'Agenda 2030 per lo sviluppo sostenibile", il Parlamento europeo ha adottato la Risoluzione sull'iniziativa sull'efficienza sotto il profilo delle risorse: ridurre lo spreco alimentare, migliorare la sicurezza alimentare (Risoluzione del 16 maggio 2017), aggiornando gli obiettivi in materia di contrasto allo spreco di cibo, già indicati nella precedente Risoluzione del 2012 (Risoluzione del 19 gennaio 2012 su Come evitare lo spreco di alimenti: strategie per migliorare l'efficienza della catena alimentare nell'UE), in risposta all'obiettivo 12.3 del documento Agenda 2030. In particolare, a fronte delle stime delle Nazioni Unite che riferiscono dell'incremento della popolazione mondiale che patisce la fame, il documento Agenda 2030 individua l'obiettivo di ridurre del $50 \%$ entro il 2030 gli sprechi alimentari globali pro capite a livello di vendita al dettaglio e di consumatori e di ridurre le perdite alimentari lungo le catene di produzione e di approvvigionamento, ivi comprese le perdite durante la fase della produzione primaria, del trasporto e dello stoccaggio.

Si intende concentrare l'attenzione sulle azioni intraprese dall'Unione europea al fine del raggiungimento dell'obiettivo di riduzione degli sprechi alimentari, con particolare riguardo alla riduzione delle perdite di cibo lungo la fase della produzione primaria. L'intervento normativo europeo sulla riduzione degli sprechi alimentari con particolare riguardo alla fase della produzione primaria dovrà necessariamente confrontarsi con le problematiche specifiche delle imprese agricole operanti lungo la filiera, come la specifica debolezza contrattuale di queste ultime che caratterizza i rapporti negoziali con le imprese acquirenti dei prodotti agricoli. Le soluzioni normative legate alla diminuzione delle perdite alimentari lungo la fase primaria non potranno, infatti, essere limitate al ricorso alle donazioni alimentari; a ben guardare, la riduzione dello spreco lungo la fase della produzione primaria è strettamente connesso alla più ampia problematica dell'equa remunerazione del produttore agricolo (art. 39 TFUE) in considerazione dell'evidente riflesso che le perdite di prodotto agricolo perfettamente commercializzabile hanno sulla remunerazione dell'attività agricola stessa. 


\section{Manuel Ernesto Montiel Silva}

\section{Desafíos del productor nicaragüense en el mercado agrícola (regional y local) y alternativas de solución. Parte 1}

\section{Introducción}

El Sistema de Integración Centroamericana - SICA - dispone de un sistema jurídico completo y complejo para la agricultura en la región. Este, a su vez, se acopla en un todo orgánico con los sistemas jurídicos de cada uno de los Estados integrantes. Entre otros aspectos importantes, el aludido sistema devela que la actividad agrícola y la comercialización de sus productos y de productos para la agricultura a nivel regional y local se desenvuelve dentro de la libertad de empresa y el libre mercado. Para que esto sea posible, entran en la dinámica de intercambio los sujetos del mercado agrícola que conforman la cadena de suministro agroalimentaria, dentro de la cual se encuentra el productor.

Se constató que esta cadena y los productores agrícolas en particular, procuran y hacen posible el crecimiento de la actividad agrícola nicaragüense. No obstante, en la práctica se percibe que la mayoría de los productores nicaragüenses enfrentan dificultades en la recuperación de los costos de producción y en la obtención de ganancias al momento de colocar sus productos en el mercado agrícola nacional. En cambio, los proveedores, los manipuladores, la agroindustria, los distribuidores, los exportadores y los especuladores se quedan con mejores réditos. Algo que invita a pensar que en la cadena de suministro agroalimentaria nacional existe una desigual distribución de los recursos generados por la actividad agrícola. 
Se considera que el problema expuesto y contextualizado exige una respuesta holística que involucra a la política, a la economía y al derecho entre otras ciencias sociales para resolverlo. Es decir, somos conscientes de que abordar el fenómeno requiere la intervención y coordinación entre varias ciencias y pretender resolverlo únicamente a través de la presente investigación sería negativamente presuntuoso.

No obstante, la viabilidad de la investigación se aloja en que problema planteado se ve inserto y disperso en la dinámica jurídica del plexo normativo que regula al mercado agrícola y a la cadena de suministro agroalimentaria, y esto es algo que permite su estudio desde la ciencia jurídica, en particular desde el derecho agrario.

La búsqueda en la doctrina nacional de trabajos que aborden el fenómeno aludido desde el punto de vista jurídico, comprueba su nula disponibilidad. En cambio, desde las ciencias de la administración, economía e ingeniería industrial, si fue posible encontrar estudios del fenómeno del mercado y de la cadena de suministro que, por cierto, fueron orientadores para la presente investigación. Más allá, en la doctrina ius agraria internacional fue posible encontrar abundantes estudios jurídicos acerca del mercado agrícola y la comercialización de productos vegetales y animales, que de igual forma fueron orientadores.

Desarrollar un estudio del fenómeno del mercado agrícola y de la cadena de suministro agroalimentaria en el ámbito del derecho coadyuvaría, por un lado, a que las demás ramas de las ciencias sociales, en el afán de dar respuestas al fenómeno del mercado y sus inequidades, consideren aspectos importantes que se extraen de la realidad jurídica. Por otro lado, sería una propuesta inicial en el contexto jurídico nacional, de lo que se debe hacer en virtud de fortalecer a los productores agrícolas en el intercambio dentro de la cadena de suministro agroalimentaria.

La conjetura o punto de partida de esta investigación fue que el marco jurídico regulador del mercado agrícola nicaragüense resulta inadecuado e insuficiente para proteger al productor agrícola en las relaciones comerciales dentro de la cadena de suministro agroalimentaria, lo que, a su vez, constituiría una eventual causa de la desigual distribución de los recursos económicos generados por la actividad agrícola, entre los diferentes actores de la cadena.

Considerando lo previo expuesto, podría mencionarse que el objetivo general planteado fue determinar las brechas dentro del marco jurídico regulador del mercado agrícola regional y nacional que permiten suprimir la desigual distribución de los recursos económicos en la cadena de suministro agroalimentaria generados por la actividad agrícola, en cuya base encontramos al productor nicaragüense. 
Es importante señalar que los desafíos jurídicos del productor agrícola nicaragüense en el mercado agrícola regional y local están vinculados a aquellos aspectos o circunstancias que le son adversas, como la debilidad del sistema que allana el camino para la desigual distribución de los recursos económicos.

En base a lo previamente expuesto, la presente investigación es de tipo teórico, debido a que se desarrolla sobre un objeto abstracto que no se puede percibir sensorialmente. Este objeto es el marco jurídico regulador del mercado agrícola centroamericano y nicaragüense y de la cadena de suministro agroalimentaria nacional.

Para comprobar la hipótesis planteada se recurrió a los métodos de análisis-síntesis, sistémico-estructural-funcional, abstracto-concreto e inductivo-deductivo. Estos permitieron determinar qué es el mercado agrícola y qué es una cadena de suministro agroalimentaria desde el punto de vista jurídico, qué elementos les componen, cómo están ordenados, cuál es su funcionamiento, cuál es la estructura jurídica que les erige y de dónde ésta surge. Paralelamente, estos métodos permitieron buscar vacíos, antinomias, confluencias, dicotomías o cualquier otra imperfección que permitiera visualizar una brecha en el sistema develado.

Para la obtención de la información y su registro se consultaron diversos recursos bibliográficos disponibles en las bibliotecas de la Universidad de Bari Aldo Moro y de la Universidad Centroamericana; los disponibles en las bases de datos de acceso remoto y de libre acceso que contienen un universo de libros, revistas indexadas y especializadas, ensayos y artículos científicos. Para el registro de la información que se ha utilizado en la fundamentación se puso en práctica el uso de fichas bibliográficas y de contenido y, adicionalmente, la elaboración de esquemas y resúmenes en cuadernos de notas. Todo ello permitió una clasificación de los argumentos por temáticas para su discusión y contrastación con la realidad. Una vez fichada la información, se procedió al análisis del contenido de las fichas bibliográficas y de los apuntes, lo que, a su vez, permitió clasificar, validar y contrastar la información para su utilización en este artículo.

\section{Marco jurídico regulador del mercado agrícola regional y local}

Para comprender qué es el marco jurídico regulador del mercado agrícola regional y local fue necesario identificar conceptos de mercado en general ${ }^{1}$

${ }^{1}$ El mercado en general no existe en realidad, sino tantos mercados como tipos de productos o servicios en tanto están organizados por un estatuto particular. Además, los mercados están interrelacionados entre sí, lo mismo que las empresas, de ahí lo importante que es no perder de vista una visión de conjunto o sistemática. Véase J. Fernández, M. García, J. Monereo, M. Morón, 
que orientaran a la comprensión del fenómeno. De este modo se hizo posible observar que el concepto de mercado es uno de los más importantes en la historia de las ciencias sociales y ha tenido un profundo impacto en sus estructuras ${ }^{2}$; más allá de las ocho definiciones que ofrece el diccionario de la Real Academia Española en línea ${ }^{3}$ y de lo que nuestro propio ordenamiento jurídico puede aportar ${ }^{4}$. Se viene formando desde hace décadas y ha sido objeto de estudio de otras ramas de las Ciencias Sociales como la Economía, algo que se demuestra al considerar los trabajos de Alejandro $\mathrm{Nadal}^{5}$, Marcela Astudillo ${ }^{6}$ y de Orlando Núñez ${ }^{7}$.

Para Alejandro Nadal, el mercado es un concepto que no ha podido ser construido de manera rigurosa por la economía, por lo que corresponde a las demás ciencias sociales - como al derecho agrario - la tarea de abordar el fenómeno del mercado ${ }^{8}$. No obstante, existen diversas conceptualizaciones de mercado y entre ellas, la que Marcela Astudillo expone al decir que la palabra mercado (...) refiere a (...) cualquier acuerdo para que compradores y vendedores de una

J. Navarro, Introducción del derecho agrario. Régimen jurídico de las explotaciones agrarias, España 2005, p. 565.

2 Véase A. Nadal, El concepto de Mercado, México 2010, p. 2, online: http://conceptos. sociales.unam.mx/conceptos_final/450trabajo.pdf.

${ }^{3}$ En el Diccionario de la Real Academia Española en línea se encuentra que la palabra mercado refiere a: 1) Contratación pública en lugar destinado al efecto y en días señalados, 2) sitio público destinado permanentemente, o en días señalados, para vender, comprar o permutar bienes o servicios, 3) concurrencia de gente en un mercado, 4) conjunto de actividades realizadas libremente por los agentes económicos sin intervención del poder público, 5) conjunto de operaciones comerciales que afectan a un determinado sector de bienes, 6) plaza o país de especial importancia o significación en un orden comercial cualquiera, 7) conjunto de consumidores capaces de comprar un producto o servicio, 8) estado y evolución de la oferta y la demanda en un sector económico dado. Online: $\mathrm{http}: / /$ dle.rae.es/?id=OyRtG0r.

${ }^{4}$ Es oportuno hacer mención que, dentro del ordenamiento jurídico nicaragüense, propiamente dentro de los Códigos Civil y Comercial, no hay disponible un concepto de mercado en general. Aunque si fue posible encontrar una definición de "mercado relevante". De acuerdo al artículo 3 de la Ley N. ${ }^{\circ} 601$, Ley de Promoción de la Competencia, el mercado relevante es la línea del comercio en una determinada zona geográfica, abarcando todos los productos o servicios razonablemente sustituibles entre sí, así como los competidores inmediatos, a los que un mayorista, intermediario o consumidor pudiese acudir a corto plazo. Véase Ley N. ${ }^{\circ}$ 601, Ley de Promoción de la Competencia, con sus reformas integradas y su Reglamento, publicada en La Gaceta Diario Oficial 206 del 24 de octubre de 2006, online: http://procompetencia.gob.ni/wp-content/uploads/2015/03/ Ley-No.601-y-Reglamento-Refundida-20161.pdf.

5 Véase la Nota 2.

${ }^{6}$ Véase M. Astudillo, Fundamentos de Economía, México 2012.

7 Véase O. Núñez, El metabolismo del mercado: Regulación-socialización-desmercantilización, Nicaragua, Anamá 2015.

${ }^{8}$ Véase la Nota 2. p. 21 
mercancía determinada hagan negocios entre sí ${ }^{9}$. La misma autora adiciona que el mercado puede concebirse como un lugar específico, en donde se reúnen e interactúan los sujetos del mercado de un sector de la economía, como el sector de productos agrícolas; o bien pueden ser grupos de personas dispersas por todo el mundo, pero relacionadas a través de internet, el teléfono o el fax, como en el caso del comercio electrónico ${ }^{10}$. Por otro lado, y en palabras del nicaragüense Orlando Núñez, el mercado, tal como existe ahora (...) es el conjunto de relaciones de intercambio de mercancías, a precios o expresiones en dinero del valor de cada una de ellas ${ }^{11}$.

En base a los conceptos expuestos, análogamente podría decirse que el mercado agrícola es - más que un lugar específico sin prescindir completamente de él - el conjunto de relaciones intersubjetivas para el intercambio de productos vegetales y animales en su estado natural o previa una o múltiples transformaciones, y de insumos, equipos, utensilios y maquinarias para la producción, a precios o expresiones en dinero del valor de cada uno de ellos. Ahora bien, esta definición permite su interpretación desde el ámbito jurídico, sin embargo, es válido aclarar a qué refiere la expresión "conjunto de relaciones de intercambio" con el particular objetivo de determinar lo que se regula jurídicamente.

De la observación se deduce que refiere al conjunto de actividades que deben realmente ejecutarse por los sujetos del mercado agrícola ${ }^{12}$ como la compra, venta, importación y exportación, para que efectivamente ocurra el intercambio de productos vegetales y animales y de productos para la agricultura. Se tiene a bien decir que estas actividades se derivan de los procesos de abastecimientos, de producción, de manipulación, de transformación, de distribución y de comercialización de estos productos, dentro o fuera de determinados territorios. Se observa que aludidas actividades de intercambio se encuentran sujetas a regulaciones normativas que son aplicables bien dentro de un Estado o bien dentro de un conjunto de Estados e incluso a nivel internacional, en base al respeto del fundamental principio de soberanía nacional.

Pero, cabe preguntar ¿de dónde surgen referidas regulaciones normativas? Se encontró que se surgen a través de acuerdos intergubernamentales y/o inte-

${ }^{9}$ Véase la Nota 6, p. 55.

${ }^{10}$ Ibídem.

${ }^{11}$ Véase la nota 7, p. 49. Cabe rescatar que este autor percibe al mercado como un organismo social y económico supremo (...) que sirve de pedestal para diferentes tipos de intercambio que conforman y caracterizan al sistema capitalista de producción, reproducción, repartición y distribución de mercancías.

${ }^{12}$ Cuando se habla de sujeto del mercado agrícola se hace referencia particularmente a los productores agrícolas, los proveedores de semillas, agroquímicos, equipos, utensilios, maquinarias y repuestos, los agro-industrializadores y comerciantes de productos agrícolas transformados o no, dentro de lo que sería una cadena de suministro corta. 
restatales. Es decir, en instrumentos jurídicos internacionales suscritos por los Estados interesados en la regulación de dicho intercambio por su trascendental importancia para el desarrollo económico de cada uno de ellos ${ }^{13}$.

Basado en lo anterior, podemos decir que el mercado agrícola centroamericano refiere a un plexo normativo devenido de instrumentos jurídicos internacionales, suscritos por los Estados que conforman al Sistema de Integración Centroamericana - SICA ${ }^{14}$ para regular el intercambio de productos vegetales y animales en su estado natural o previa una o múltiples transformaciones y de productos para la agricultura ${ }^{15}$. De aquí se desprende que el marco jurídico regulador del mercado agrícola regional equivale al plexo normativo devenido de los tratados de integración, tratados de libre comercio entre la región y otros países, el acuerdo de asociación con la Unión Europea y las Resoluciones Ministeriales Hemisféricas en el marco de la Organización de Estados Americanos - OEA ${ }^{16}$. Estos instrumentos jurídicos supranacionales, ciertamente, regulan el intercambio de productos agrícolas y de productos para la agricultura en el espacio regional integrado por El Salvador, Guatemala, Belice, Honduras, Nicaragua, Costa Rica y Panamá, todos ellos miembro del SICA ${ }^{17}$.

Por abstracción, el marco jurídico regulador del mercado agrícola nicaragüense equivaldría al plexo normativo integrado por la Constitución, las leyes,

${ }^{13}$ En la actualidad se percibe la existencia de nuevas y poderosas fuentes de producción normativa de carácter supraestatal, interestatal y paraestatal (...) determinado por la existencia de poderes normativos superiores al Estado como lo son los organismos internacionales o supranacionales. Véase J. Pérez, Las fuentes del derecho y el derecho agrario cubano, Madrid 2015, p. 43.

${ }^{14}$ Véase Sistema de Integración Centroamericana - SICA, Protocolo de Tegucigalpa a la Carta de Organización de Estados Centroamericanos - ODECA, (Honduras, Presidentes de Costa Rica, El Salvador, Guatemala, Honduras, Nicaragua y Panamá, 1991), online: http://www.sice. oas.org/trade/sica/SG121391.asp.

${ }^{15}$ El mercado en el sentido jurídico es (...) la unidad legal de las relaciones de intercambio. Véase A. Germanò, E. Rook Basile, Diritto agrario. Trattato di diritto privato dell'Unione Europea, Torino 2006, p. 191.

${ }^{16}$ Véase Acuerdos Ministeriales Hemisféricos - AMH, Plan Agro 2003-2015 para la Agricultura y Vida Rural en las Américas y Declaración de Bávaro, San José, Instituto Interamericano de Cooperación para la Agricultura - IICA, 2010, p.133, online: http://repiica.iica.int/docs/B1662e/ B1662e.pdf.

${ }^{17}$ Centroamérica es una Comunidad de Estados económico-política cuyas relaciones se enmarcan en un esquema de integración regional que no es puramente económica, sino global abarcando los diversos sectores económicos y políticos pero también social, cultural y ambiental, cuyos presupuestos se centran en una concepción social y democrática del Estado en el marco irrestricto de la tutela, respeto y promoción de los Derechos Humanos, debiendo los Estados miembros (del SICA) comportarse de acuerdo a los principios fundamentales contenidos en el artículo 4 del "Protocolo de Tegucigalpa". Véase en O. Guerrero, El derecho comunitario: concepto, naturaleza y caracteres, "Revista de Derecho de la Universidad Centroamericana - UCA" 2003, Núm. 6, pp. 208-209, online: http://www.revistasnicaragua.net.ni/index.php/revderecho/article/view/937. 
decretos, normas técnicas y resoluciones administrativas que regulan y garantizan el intercambio de productos agrícolas y de productos para la agricultura dentro del territorio de la República de Nicaragua.

\section{Marco jurídico regulador del mercado agrícola regional}

En este espacio es oportuno rescatar que el plexo normativo inherente al intercambio de productos agrícolas en la región centroamericana se acopla al sistema jurídico completo y autosuficiente para la agricultura en el ámbito jurídico internacional ${ }^{18}$. Se ve directamente influenciado por instrumentos jurídicos internacionales de mayores alcances, como el Acuerdo de Marrakech ${ }^{19}$ y los acuerdos comerciales multilaterales anexos a él, en particular, los acuerdos siguientes: Acuerdo sobre Aranceles Aduaneros y Comercio de 1994, Acuerdo de Agricultura, Acuerdo sobre Aplicación de Medidas Sanitarias y Fitosanitarias y el Acuerdo sobre Obstáculos Técnicos al Comercio. Del mismo modo, sobre él ejerce influencia el Plan Agro 2003-2015 como instrumento jurídico hemisférico adoptado en la Segunda Reunión Ministerial "Agricultura y Vida Rural en las Américas", celebrada en la Ciudad de Panamá en el año 2003 y que encuentra respaldo para su aplicación en el Mandato de la Cumbre Extraordinaria de las Américas celebrada en la Ciudad de Monterrey (México) en el año 2004²0.

Este marco jurídico se ve integrado por el Protocolo de Tegucigalpa de 1991 a través del cual fue conformado el SICA ${ }^{21}$; y el Protocolo al Tratado General de Integración Económica Centroamericana (Protocolo de Guatemala) de 199322,

${ }^{18}$ El pasaje del GATT a la OMC significó (...) la constitución de un verdadero sistema jurídico completo y autosuficiente (...) para la agricultura internacional. Véase L. Pastorino, La agricultura en el ámbito internacional. Aspectos jurídicos sobresalientes de los acuerdos de la OMC y sus proyecciones concretas en la actividad agraria y los mercados agropecuarios, España 2008, p. 101.

${ }^{19}$ La Declaración de Marrakech es considerada el marco jurídico más fuerte y más claro que han adoptado para el desarrollo del comercio internacional y que incluye un mecanismo de solución de diferencias más eficaz y fiable; la reducción global de los aranceles en un $40 \%$ y los acuerdos más amplios de apertura de los mercados en el sector de las mercancías, el establecimiento de un marco multilateral de disciplinas para el comercio de servicios y para la protección de los derechos de propiedad intelectual relacionados con el comercio, así como el reforzamiento de las disposiciones multilaterales sobre el comercio de productos agropecuarios y de textiles y prendas de vestir. Véase Organización Mundial del Comercio - OMC, Acuerdo de Marrakech por el que se establece la Organización Mundial del Comercio, Marruecos, Ministros de 124 Gobiernos, 1994, en línea. Online: https://www.wto.org/spanish/docs_s/legal_s/04-wto_s.htm.

${ }^{20}$ Véase Nota 16.

21 Véase Nota 14.

22 Véase Gobiernos de las Repúblicas de Costa Rica, El Salvador, Guatemala, Honduras, Nicaragua y Panamá, Protocolo al Tratado General de Integración Económica Centroamericana 
a través de la cual los Estados se comprometieron en perfeccionar la zona de libre comercio para todos los bienes originarios de sus respectivos territorios, a través de la eliminación gradual de las barreras arancelarias y no arancelarias al comercio intrarregional, eliminando toda restricción de carácter cuantitativo y cualquier otra medida de efecto equivalente.

Consecuentemente por el Régimen Arancelario y Aduanero Centroamericano, que, a su vez, se ve integrado por el Arancel Centroamericano de Importación, el Acuerdo para la aplicación del artículo VII del Acuerdo General sobre Aranceles Aduaneros y Comercio - GATT 1994, el Código Aduanero Uniforme Centroamericano - CAUCA y su reglamento RECAUCA ${ }^{23}$. Igualmente lo integra la Política Agrícola Centroamericana 2008-201724, que dentro de sus objetivos estratégicos se encuentran: la prosperidad rural, la seguridad alimentaria, el posicionamiento internacional, la competitividad, la sustentabilidad, la equidad y la gobernabilidad.

También forman parte de aludido marco los Tratados de Libre Comercio suscrito entre Centroamérica y los siguientes países: República Dominicana el 16 de abril de 1998, Chile el 18 de octubre de 1999, Estados Unidos el 5 de agosto de 2004, Panamá el 15 de enero de 2009, México el 22 de noviembre de 2011; Taiwán el 16 de junio de 2006 y el Acuerdo de Asociación con la Unión Europea el 29 de junio de $2012^{25}$. De la revisión estructural de estos instrumen-

(Protocolo de Guatemala), Guatemala, 1993, online: http://www.sice.oas.org/Trade/sica/PDF/ Prot.Guatemala93.pdf.

${ }^{23}$ Véase Secretaria de Integración Económica Centroamericana - SIECA, Proceso de la integración de Panamá a la Integración Económica Centroamericana. Temas arancelarios. Guatemala: Autor, 2011, p. 3, online: https://www.mici.gob.pa/imagenes/pdf/presentacion_arancelaria_panama. pdf.

${ }^{24}$ Uno de los temas transversales de la Política Agrícola Centroamericana es el que corresponde a la pequeña agricultura empresarial. Entre otras cosas dispone que se debe promover la inserción de la pequeña agricultura en los mercados, así como su integración regional, fortalecimiento organizacional y asociatividad, véase Consejo Agropecuario Centroamericano, Política Agrícola Centroamericana 2008-2017, Costa Rica, Consejo Agropecuario Centroamericano, 2007, p. 73, online: http://www.fao.org/forestry/13772-0e3d01f7a6aa2707e127bf0bc4d796edb.pdf.

${ }^{25}$ Véase Sistema de Información sobre Comercio Exterior - SICE, Tratado de Libre Comercio entre Centroamérica y República Dominicana, República Dominicana, Presidentes de Costa Rica, Honduras, República Dominicana, El Salvador, Guatemala y Nicaragua, 1998.

: http://www.sice.oas.org/Trade/camdrep/CARdo_2s.asp\#20.01; Sistema de Información sobre Comercio Exterior - SICE, Tratado de Libre Comercio entre Centroamérica y Chile, Managua, 1999, online: http://www.sice.oas.org/Trade/chicam/chicamin.asp; Sistema de Información sobre Comercio Exterior - SICE, Tratado de Libre Comercio entre Centroamérica, Estados Unidos y República Dominicana, Washington 2004, online: http:/www.sice.oas.org/Trade/CAFTA/CAFTADR/ CAFTADRin_s.asp; Sistema de Información sobre Comercio Exterior, Tratado de Libre Comercio entre Centroamérica y Panamá, Panamá 2009, online: http://www.sice.oas.org/Trade/capan/indice. asp; Sistema de Información sobre Comercio Exterior - SICE, Tratado de Libre Comercio entre 
tos se desprende un patrón normativo encaminando al establecimiento de una zona de libre comercio y todo lo que ello implica, como la libre circulación de la mercancía, la desgravación arancelaria, la identificación y el reconocimiento de los productos originarios, el trato nacional de los mismos, la competencia leal, el libre acceso de bienes al mercado. Sin olvidar reglas especiales para los productos agrícolas, como los acuerdos sanitarios y fitosanitarios y lo referente a los obstáculos técnicos al comercio también establecidos en ellos.

Se puede agregar que estos instrumentos jurídicos supranacionales surgieron de la necesidad de los Estados centroamericanos de alcanzar el desarrollo económico y social sostenible, en el que la agricultura desempeña un papel muy importante, por ser fuente tradicional de empleo y generadora de alimento para la población, con marcada incidencia en el crecimiento del Producto Interno Bruto - PIB ${ }^{26}$.

Finalmente, en lo que respecta a este acápite se puede parcialmente concluir que todos los instrumentos jurídicos internacionales en este acápite referidos sirven de base para visualizar al marco jurídico regulador del mercado agrícola centroamericano con marcado interés en incentivar zonas de libre comercio; y sirven además para promover una política común en el sector agrícola.

\section{CHALLENGES FACING A NICARAGUAN PRODUCER OPERATING IN THE AGRICULTURAL MARKET (REGIONAL AND LOCAL) AND ALTERNATIVE METHODS OF SOLVING THEM. PART 1}

\section{Sum mary}

The aim of the article is to present the regulation of the Nicaraguan agricultural market and to identify legal solutions that could contribute to overcoming the unequal distribution of economic resources resulting from domestic agricultural activity in the food supply chain. In particular, the aim is to identify the legal loopholes that contribute to the unequal distribution of resources in the Nicaraguan agri-food supply system and to propose alternative solutions that are available from the point of view of agricultural law science.

In the author's opinion, the main difficulty facing the Nicaraguan agricultural producer is the commercialisation of own goods on the agricultural products market of Central America and Nicaragua and the regulatory deficiencies in this respect. The System of Central American

México y Costa Rica, El Salvador, Guatemala, Honduras y Nicaragua, San Salvador 2011, online: http://www.sice.oas.org/Trade/CACM_MEX_FTA/Index_s.asp; Sistema de Información sobre Comercio Exterior - SICE, Tratado de Libre Comercio entre Nicaragua y China (Taiwan), Taipei 2006 online: http://www.sice.oas.org/Trade/NIC_TWN/NIC_TWN_s/index_s.asp; Sistema de Información sobre Comercio Exterior, Acuerdo de Asociación Centroamérica y Unión Europea, Tegucigalpa 2012,. online: http://www.sice.oas.org/Trade/CACM_EU/Text_Sept14/Index_s.asp.

${ }^{26}$ Véase Nota 24, p. 17. 
Integration (Sistema de la Integración Centroamericana - SICA) in force in most countries of the region, includes on the one hand comprehensive legal regulations concerning agriculture, but on the other hand it does not fully correspond to the modern structure of the agri-food chain. In practice, regional and national legislation is subject to frequent amendments and provides unsatisfactory protection for agricultural producers at every stage of production. The introduction of changes at the regional level in the form of free economic circulation zones and the implementation of the common agricultural policy by Central American countries offers a certain alternative to this situation.

\title{
SFIDE DEL PRODUTTORE NICARAGUENSE NEL MERCATO AGRICOLO (REGIONALE E LOCALE) E SOLUZIONI ALTERNATIVE. PARTE 1
}

\author{
Riassunto
}

Lo scopo dell'articolo è di presentare la regolamentazione del mercato agricolo in Nicaragua e di indicare soluzioni giuridiche che potrebbero contribuire a superare una distribuzione iniqua delle risorse economiche derivanti dall'attività agricola nazionale all'interno della filiera alimentare. In particolare, si tratta di individuare scappatoie giuridiche che contribuiscono ad una distribuzione iniqua delle risorse nel regime nicaraguense di approvvigionamento per i prodotti agroalimentari e di proporre soluzioni alternative per la loro eliminazione alla luce della scienza del diritto agrario.

Secondo l'autore, la principale difficoltà per il produttore agricolo nicaraguense è il processo di commercializzazione dei prodotti sul mercato dei prodotti agricoli dell'America centrale e del Nicaragua nonché carenze normative in questo ambito. Il Sistema dell'integrazione centroamericana (SICA), vincolante nella maggior parte dei paesi della regione, da un lato contiene regolazioni giuridiche complete sull'agricoltura, dall'altro non corrisponde pienamente alla struttura moderna della filiera agroalimentare. In pratica, la legislazione regionale e nazionale è soggetta a frequenti cambiamenti e non protegge in modo sufficiente il produttore agricolo in ogni fase di produzione. Un'alternativa sarebbe quella di introdurre cambiamenti a livello regionale, sotto forma di aree di libero scambio e di attuare la politica agricola comune da parte dei Paesi dell'America centrale. 